\title{
Experimental Classification and Enhanced Suppression of Parasitic Oscillations in Gyrotron Beam Tunnels
}

\author{
Zisis C. Ioannidis, loannis Chelis, Gerd Gantenbein, Tomasz Rzesnicki, \\ and John Jelonnek, Senior Member, IEEE
}

\begin{abstract}
High-power gyrotrons may suffer from parasitic oscillations that are excited in the electron-beam compression zone. Different damping structures are proposed in the literature that reduce the possibility of parasitic excitation by increasing the starting currents of the modes. In this work we focus on a dielectric-loaded (stacked) beam tunnel. Based on our previous theoretical studies, we make targeted modifications to the beam tunnel in order to classify the parasitic signals and localize the areas where they are excited. After two successive modifications, the beam tunnel exhibits improved behavior with higher starting currents of the parasitic modes. The experiments are performed by using a modular $170 \mathrm{GHz}, 1 \mathrm{MW}$ short-pulse gyrotron, which due to its flanged construction gives the possibility to modify the beam tunnel without affecting the rest of the tube.
\end{abstract}

Index Terms-Beam tunnel, dielectric-loaded, gyrotron, parasitic oscillations

\section{INTRODUCTION}

$\mathrm{G}$ YROTRONS are the only high-frequency, high-power millimeter-wave source that is able to cover the Electron Cyclotron Resonance Heating (ECRH) and Electron Cyclotron Current drive (ECCD) needs of modern fusion experiments. There are already numerous examples of successful ShortPulse (SP) and Continuous-Wave (CW) gyrotrons that cover the frequency range from $100 \mathrm{GHz}$ to $200 \mathrm{GHz}$ with demonstrated RF power up to $2 \mathrm{MW}$ and a total efficiency that in depressed collector operation can exceed 50\% [1].

In several recent experiments with MW-class gyrotrons, the tubes suffered from undesired parasitic oscillations that were excited in the region of the beam tunnel [2]-[4]. Such

Manuscript received August 24, 2020; revised September 14, 2020; accepted September 16, 2020. This work has been partly carried out within the framework of the EUROfusion Consortium and has received funding from the Euratom research and training programme 2014-2018 and 2019-2020 under grant agreement No 633053. The use of the Short Pulse Gyrotron owned by Fusion for Energy is managed through F4E-GRT-553. The views and opinions expressed herein do not necessarily reflect those of $\mathrm{F} 4 \mathrm{E}$ and the European Commission.

Z. Ioannidis, G. Gantenbein, T. Rzesnicki and J. Jelonnek are with the Institute for Pulsed Power and Microwave Technology (IHM) at the Karlsruhe Institute of Technology (KIT), Germany (email: zisis.ioannidis@kit.edu).

I. Chelis is with the Physics Department of the National and Kapodistrian University of Athens, Zografou, Greece. problems have also been reported in older experiments [5], [6]. These parasitic oscillations are a significant threat for the efficient gyrotron operation, since they can reduce the quality of the electron beam (due to induced spreads) before the beam-wave interaction takes place in the cavity. As a result, the output RF power and the efficiency of the gyrotron could be limited. In addition, the gyrotron may exhibit increased thermal loads at unpredicted positions and the pulse length may be reduced due to arcing or intense outgassing.

Considering that the demand for output power is constantly increasing, it would be beneficial to be able to operate modern gyrotrons at higher frequencies and with significantly higher beam currents, while minimizing the possibility to excite spurious oscillations in the beam compression area. Various alternative mode-damping structures have been proposed and developed for this reason. The proposed beam tunnels include arbitrarily non-axisymmetric corrugated metallic structures [7]-[8], conical silicon carbide ( $\mathrm{SiC})$ structures with weakly conducting $\mathrm{SiC}$ to dissipate static charges [4], as well as alternating stacks of ceramic and copper rings [9].

The stacked beam tunnels are probably the most common structures and have been successfully used in series production gyrotrons, without however suppressing the parasitic oscillations completely at higher beam currents. Such beam tunnels have been studied by using various in-house semi-analytic numerical codes [10]-[12]. Recently, detailed design guidelines were provided in order to maximize the dielectric losses and increase the starting currents of the parasitic modes [13]. In particular, it was shown that a significant increase of the absorption of the dielectric material can be achieved, provided that materials with relatively low values of the real and imaginary parts of the permittivity are used. This is in contradiction to the established approach of the past, which suggested the use of absorbing ceramics with very high loss tangent in order to increase the dielectric losses.

In this work we make targeted modifications and we study experimentally a dielectric-loaded (stacked) beam tunnel with the goal to classify the observed parasitic signals and further suppress them. In particular, in Section II we describe in detail the test bench and the diagnostics that are used for the experiments. In Section III we record the performance and compare the initial design of the beam tunnel with its modified versions; first after tailoring the properties of the dielectric 
material according to [13] and second after replacing the major part of the metallic spacer with stacked metallic and ceramic rings. In Section IV we discuss the effect of the presence of the parasitic signals on the output RF power. Our conclusions are summarized in Section V.

\section{Test Bench, Diagnostics and Measurements}

The experiments have been performed by using a modular, short-pulse, $170 \mathrm{GHz}-1 \mathrm{MW}$ conventional gyrotron [14], [15]. The pulse length is $1 \mathrm{~ms}$ for all the tests. The gyrotron can be operated with either beam current $I_{\mathrm{b}}=40 \mathrm{~A}$ and accelerating voltage $V_{\text {acc }}=80 \mathrm{kV}$ (high-voltage operating point, HVOP) or with current $I_{\mathrm{b}}=45 \mathrm{~A}$ and voltage $V_{\text {acc }}=76.6 \mathrm{kV}$ (low-voltage operating point, LVOP) [15]. Due to the modular nature of the gyrotron, it is easy to replace any component and compare the performance of the modified tube with the one of the previous design, without introducing additional uncertainties. The beam tunnel is also modular itself. It consists of a housing and the internal parts. Each time a beam-tunnel variation is tested, the housing remains the same, whereas the internal parts are replaced by the new ones.

Two diagnostic systems are used in order to identify possible parasitic oscillations. Both systems are fed with properly down-converted stray RF radiation coming out of the relief window of the gyrotron. The first system is a ninechannel filter-bank spectrometer [16] that covers the RF range $140-175 \mathrm{GHz}$ with intermediate frequencies (IF) $0.1 \mathrm{GHz}-$ $18 \mathrm{GHz}$. Fig. 1 presents a typical screenshot of the filterbank signals during a pulse. Due to the non-linear nature of the down-mixing, harmonic frequency products of the local oscillator could falsely appear as parasitic oscillations. It is not possible to identify if a signal is a spurious product of the mixing procedure by just monitoring it with the filter bank. This is not a limitation for the second system used, namely the in-house Pulse Spectrum Analysis (PSA) system [17]. This system uses two local oscillators with different frequencies in order to filter out redundant results. If a detected frequency does not appear in the spectrum of both down-converted signals, then it is considered as spurious and it is rejected by the software. Summarizing, for every operating point tested, the spectrum range $140 \mathrm{GHz}-175 \mathrm{GHz}$ is monitored with the filter bank and then each detected signal is studied with the PSA system to decide if it is spurious or not. Additionally, the variation of the signal frequency with respect to time is monitored. We note that measuring the parasitic signals coming out of the relief window does not provide any information about the region of their excitation (pre-cavity or after-cavity oscillations). In the past, some parasitic signals were assumed to originate from after-cavity oscillations, a hypothesis which was supported by some numerical results but not confirmed experimentally [9], [18].

In order to optimize its performance, the gyrotron is, usually, operated with various magnetic field profiles. The magnetic field profile is varied in a systematic way and it is controlled by the magnetic field angle $\varphi_{\mathrm{B}}$ at the area of the

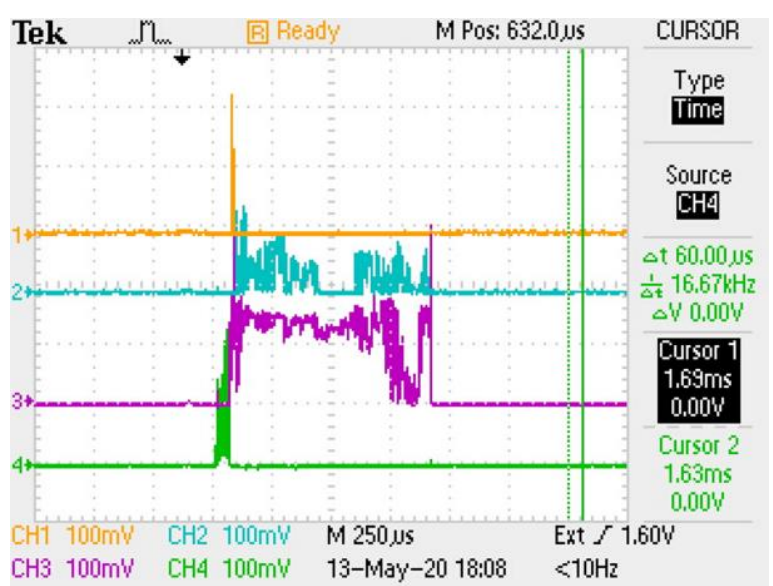

Fig. 1. Filter-bank [16] screenshot with the nominal mode (magenta) and a possibly parasitic signal (cyan).

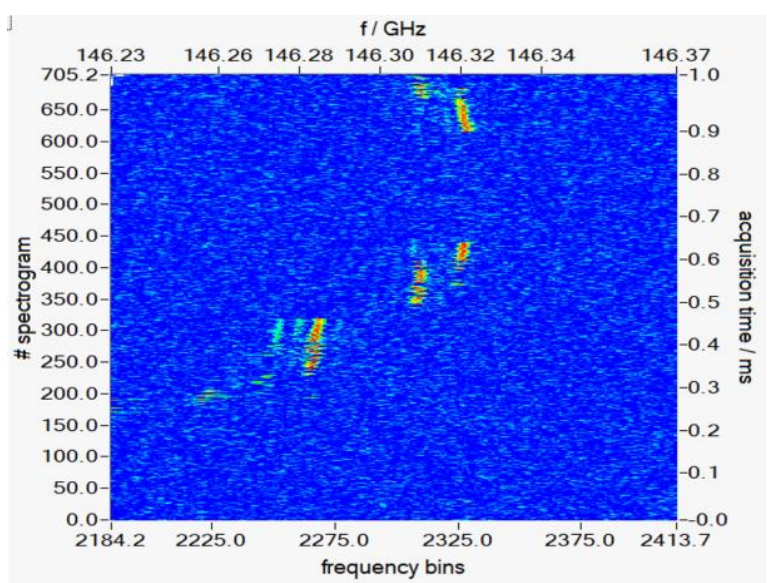

Fig. 2. Verification by the PSA system [17] that the signal detected by the filter bank is a parasitic signal with frequency $\sim 146 \mathrm{GHz}$.

emitter and the beam radius $R_{\mathrm{b}}$ at the area of the cavity. Depending on the gun design, both parameters can control the pitch factor of the electron beam, while keeping the magnetic field at the cavity constant. As a rule of thumb for the gun of the gyrotron used in the experiments, more negative magnetic field angles and smaller beam radii correspond to higher values of the pitch factor [19], provided that the accelerating voltage is kept constant. For every $\left(\varphi_{\mathrm{B}}, R_{\mathrm{b}}\right)$ combination the output power $P_{\mathrm{RF}}$ and the corresponding total efficiency $\eta_{\text {tot }}=P_{R F} / \mathrm{V}_{\text {acc }} / \mathrm{I}_{\mathrm{b}}$ (non-depressed collector operation) is recorded for the maximum optimized accelerating voltage $V_{\text {acc }}$ that can be applied before the operating mode switches to the next competitor. Due to the different accelerating voltage and the Schottky effect at the emitter, the beam current of each operating point can be slightly different within a range of a few amperes. The RF power results are presented with normalized contour plots. All the $\left(\varphi_{\mathrm{B}}, R_{\mathrm{b}}\right)$ combinations with confirmed parasitic signals by the PSA system are noted on the power contour plots with scatter points. In this way the contour plots give immediately an overview of the number of points where parasitics appear, as well as of the gyrotron performance. The frequency ranges of interest are represented on the power contour plots by different point types (squares or circles). 


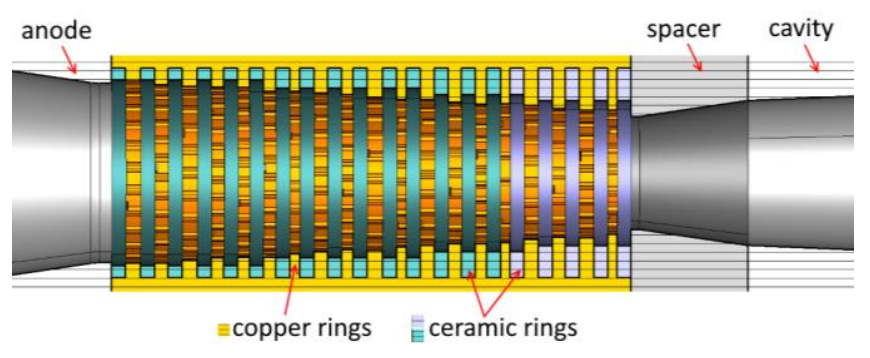

Fig. 3. Representation of a stacked beam tunnel with alternating copper (orange color) and lossy ceramic (green/purple color) rings.

\section{EXPERIMENTAL RESULTS}

\section{A. Configuration I: Standard stacked beam tunnel (high- permittivity ceramic)}

The beam tunnel that is used as the baseline in our experiments is a stacked beam tunnel, like those that are found in many European and US high-power gyrotrons [9]. In particular, the beam tunnel consists of alternating copper and lossy ceramic rings (not brazed together in our short-pulse configuration), similar to the stacked geometry presented in Fig. 3. The copper rings define the electric potential of the compression zone, whereas the ceramic rings aim to introduce losses in the structure and increase the starting currents of the parasitic modes. The beam tunnel does not cover the complete area of the compression zone and there is a smooth metallic section (usually called spacer) connecting the stacked structure with the cutoff section of the cavity (Fig. 3). The ceramic rings are made of $\mathrm{BeOSiC}$ with a relatively high concentration of $\mathrm{SiC}$ [1]. In order to increase its suppression performance, additional irregular corrugations have been introduced in the copper rings with roughly quarter-wavelength depth [9]. Fig. 4 presents the measured values for the real part of the relative permittivity $\varepsilon_{\mathrm{r}}$ and the corresponding loss tangent with respect to the frequency for the type of dielectric material used in the beam tunnel. The measurements have been performed in house at KIT by using a thin sample of the material and a WR5.1 Material Characterization Kit (MCK) [20]. According to the depicted measurements in Fig. 4, the dielectric constant has very high real and imaginary parts in the complete frequency band. On the average the complex permittivity is approximately $\varepsilon_{\mathrm{r}} \approx 22-j 17$. Note that due to the very high loss tangent of the material, it was mandatory to use a very thin sample for the measurement of the dielectric properties.

For the nominal operating parameters of the gyrotron, i.e. beam current up to $45 \mathrm{~A}$, no parasitic oscillations are detected. If the beam current is above $47 \mathrm{~A}$ we start to detect spurious signals, without a noticeable degradation of the gyrotron performance. For this reason, we focus the experiments at two beam-current levels: a lower one just above the nominal parameters and a significantly higher one. Fig. 5a presents the output RF power for a low average beam-current value, i.e. $I_{\mathrm{b}}=47 \mathrm{~A}$. The power results in the contour plot are presented in $\mathrm{dB}$ with respect to the optimal operating point, which has been found to be $\left(\varphi_{\mathrm{B}}, R_{\mathrm{b}}\right)=\left(-2^{\circ}, 9.40 \mathrm{~mm}\right)$ with output power $1.00 \mathrm{MW}$ and efficiency 26\% (in non-depressed collector

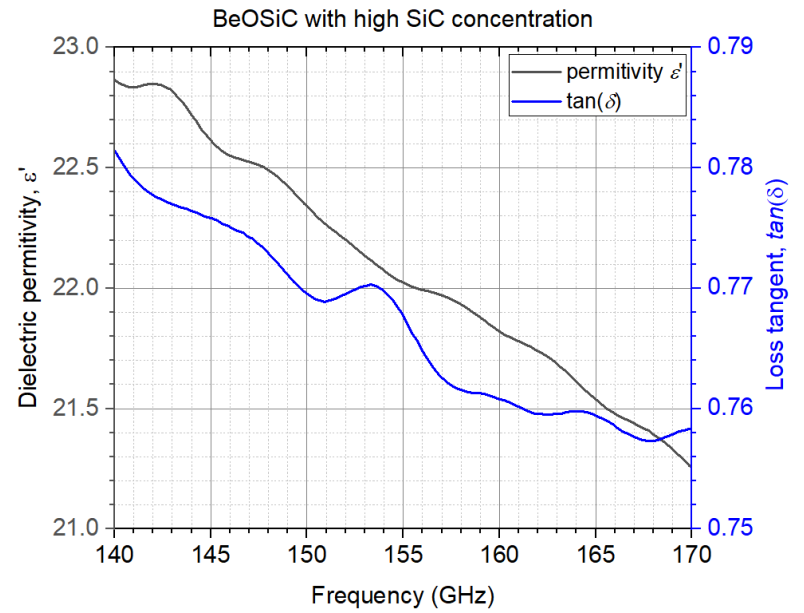

Fig. 4. Real part of the dielectric permittivity and loss tangent of BeOSiC with high $\mathrm{SiC}$ concentration.
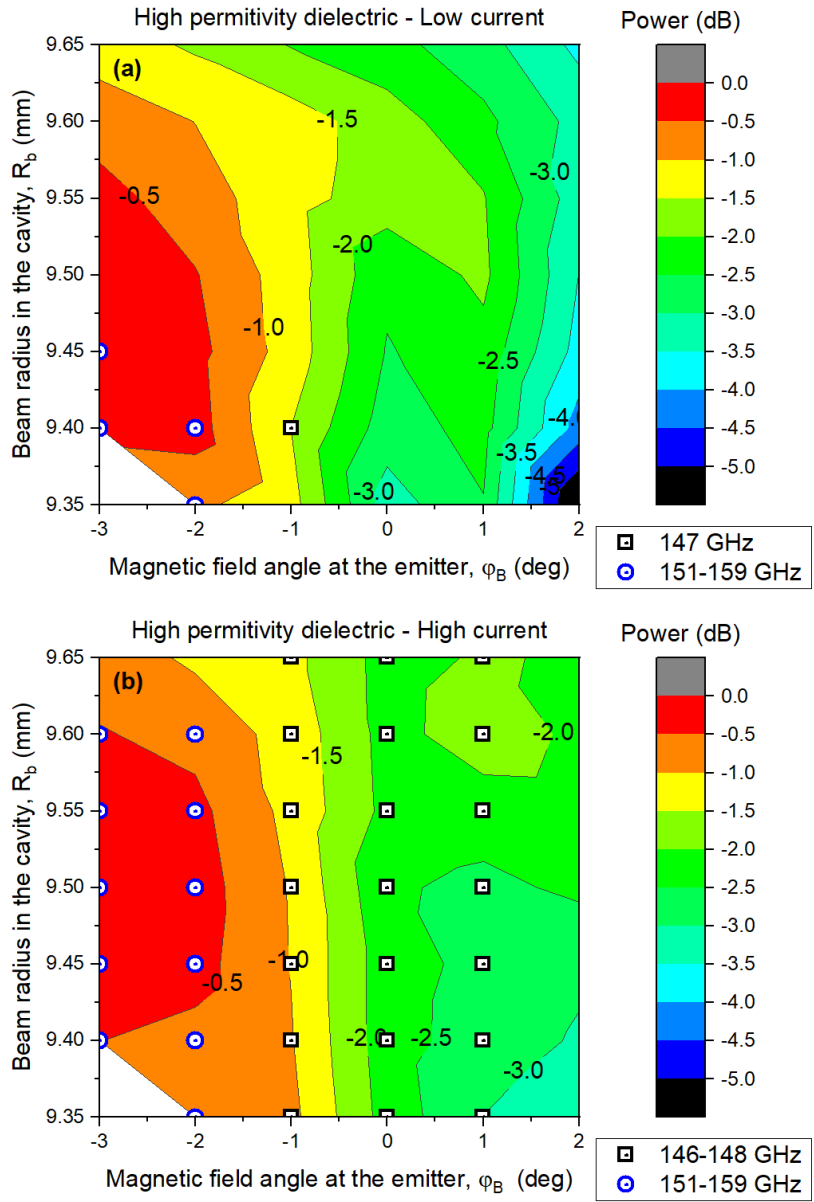

Fig. 5. Standard stacked beam tunnel with high permittivity ceramic rings. Output power with respect to the magnetic field angle $\varphi_{B}$ at the emitter and the beam radius $R_{\mathrm{b}}$ in the cavity for (a) low $(47 \mathrm{~A})$ and $(\mathrm{b})$ high $(55 \mathrm{~A})$ beam current.

operation). In the same figure, the scatter points correspond to the operating parameters were a parasitic signal has been detected. In particular, the blue circles correspond to frequencies in the range $151 \mathrm{GHz}-159 \mathrm{GHz}$, whereas the black squares indicate parasitic frequencies around $147 \mathrm{GHz}$, a distinction that is further discussed in Sections II.B and II.C. 
Evidently, for the low-current case, parasitic signals are detected only for a few operating points, where the pitch factor is expected to be rather high.

This is not the case for higher current values. Fig. 5b presents the output power for an average beam current $I_{\mathrm{b}}=55 \mathrm{~A}$. The power results are presented in $\mathrm{dB}$ with respect to the currently optimal operation, which has been achieved at $\left(\varphi_{\mathrm{B}}, R_{\mathrm{b}}\right)=\left(-3^{\circ}, 9.55 \mathrm{~mm}\right)$ with output power $1.20 \mathrm{MW}$ and efficiency $27 \%$. Now, parasitic oscillations with frequencies in the range $146 \mathrm{GHz}-160 \mathrm{GHz}$ are, essentially, detected for every tested $\left(\varphi_{\mathrm{B}}, R_{\mathrm{b}}\right)$ combination. In detail, parasitic signals with frequencies $151 \mathrm{GHz}-160 \mathrm{GHz}$ (black circles) are detected for magnetic field angle $\varphi_{\mathrm{B}} \leq-2^{\circ}$, whereas signals with frequencies $146 \mathrm{GHz}-148 \mathrm{GHz}$ (blue squares) are detected for $\varphi_{\mathrm{B}} \geq-1^{\circ}$.

\section{B. Configuration II: Stacked beam tunnel with optimized absorbers (low-permittivity ceramic)}

According to [13], a significantly lower concentration of the lossy additive in the ceramic is preferable. In brief, by reducing the concentration of $\mathrm{SiC}$, the resulting ceramic exhibits much lower permittivity values (both real and imaginary part). In turn, the potentially generated RF field in the beam tunnel area is not strongly reflected at the vacuumceramic interface and penetrates deeper inside the damping material, increasing in this way the overall losses. This is not the case for materials with very high loss-tangent values, which have a high reflection coefficient at the interface under discussion and consequently exhibit lower overall losses.

Following the design guidelines in [13], the last five ceramic rings towards the spacer (purple color in Fig. 3), were replaced by a $\mathrm{BeOSiC}$ compound with tailored permittivity. The dielectric properties of the new material were measured and as shown in Fig. 6, it had roughly three times lower values for the real part of the permittivity and one order of magnitude lower loss-tangent values. Special care was taken in order to avoid an extremely low imaginary part of the permittivity that would result in resonances inside the area of the ceramic and possibly very low absorption [13]. We modified only the last five rings since the pitch factor is higher in this region and this is where damage from parasitic oscillations has been observed in past experiments [2].

Fig. 7a presents the RF power of the gyrotron with the optimized low-permittivity ceramics for average beam current $I_{\mathrm{b}}=47 \mathrm{~A}$. The power results are normalized to the optimal performance of the gyrotron equipped with the optimized ceramics. This is achieved at the operating point $\left(\varphi_{\mathrm{B}}, R_{\mathrm{b}}\right)=(-$ $2^{\circ}, 9.40 \mathrm{~mm}$ ) where $0.97 \mathrm{MW}$ are generated with total efficiency $26 \%$. With respect to the detected parasitic signals, Fig. 7a is comparable to Fig. 5a.

The beneficial effect of the modification becomes, however, clear when the gyrotron is operated with a higher beam current. Fig. $7 \mathrm{~b}$ presents the output power for $I_{\mathrm{b}}=55 \mathrm{~A}$ normalized to the one achieved at $\left(\varphi_{\mathrm{B}}, R_{\mathrm{b}}\right)=\left(-2^{\circ}, 9.45 \mathrm{~mm}\right)$, where $1.15 \mathrm{MW}$ are generated with total efficiency $26 \%$. The slightly different performance, compared to the one achieved with the initial beam tunnel for high beam current, relies

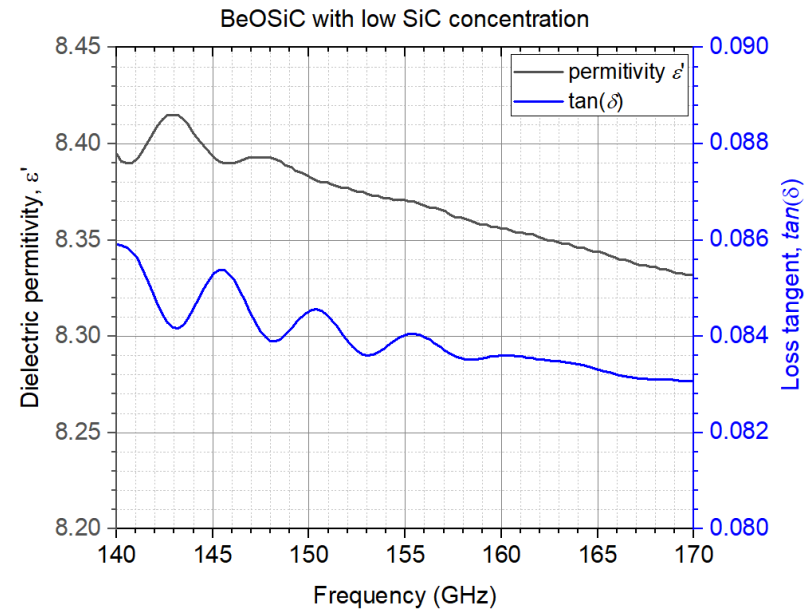

Fig. 6. Real part of the dielectric permittivity and loss tangent of $\mathrm{BeOSiC}$ with low $\mathrm{SiC}$ concentration.
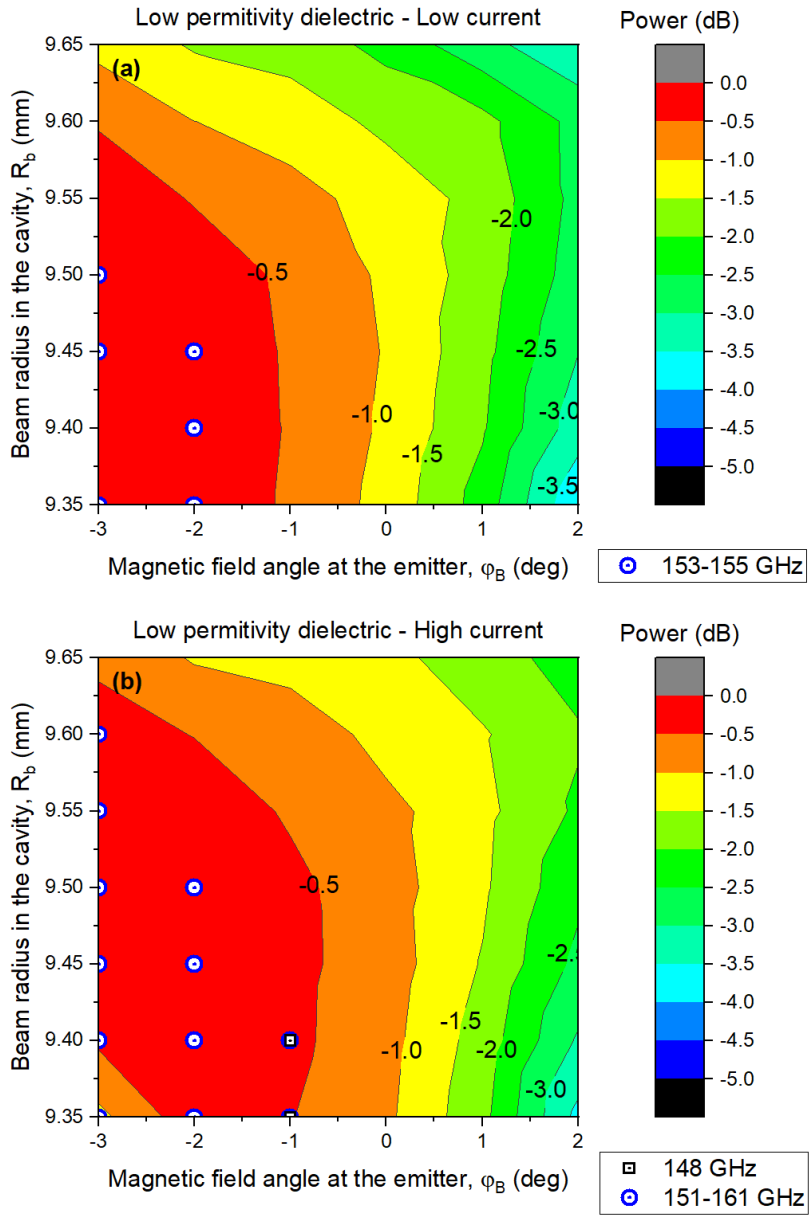

Fig. 7. Stacked beam tunnel with low-permittivity ceramics [13]. Output $\mathrm{RF}$ power with respect to the magnetic field angle $\varphi_{\mathrm{B}}$ at the emitter and the beam radius $R_{b}$ in the cavity for (a) low (47 A) and (b) high beam current $(55 \mathrm{~A})$.

within the error margins of the measurement $(5 \%$ for the power and $2 \%$ for the efficiency) and could also be related to the positioning of the gyrotron in the magnet [21] as well as to the time that the emitter had to recover after the opening of the tube for the modification of the beam tunnel. It is evident from Fig. $7 \mathrm{~b}$ that the majority of the parasitic signals have been 
suppressed. In particular, the frequencies in the range $146 \mathrm{GHz}-148 \mathrm{GHz}$ are not detected any more. This is a strong indication that these parasitic oscillations are excited at the last part of the beam tunnel, where the low-permittivity dielectric material was introduced. In parallel the high-power operation region of the gyrotron has been extended towards the positive values of the magnetic field angle (Fig. 7b).

On the contrary, the parasitic signals with frequencies higher than $151 \mathrm{GHz}$ have been only slightly affected. Considering that the cyclotron frequency at the border between the beam tunnel and the spacer is around $150 \mathrm{GHz}$, it is reasonable to assume that the $151 \mathrm{GHz}-159 \mathrm{GHz}$ signals are excited mostly in the spacer area. The strong possibility of parasitic excitation in the spacer area has been shown by means of numerical simulations in [22]. It should be noted that for the operating points $\left(-1^{\circ}, 9.40 \mathrm{~mm}\right)$ and $\left(-1^{\circ}, 9.35 \mathrm{~mm}\right)$ both types of parasitic signals are noted in Fig. 7b. However, the $148 \mathrm{GHz}$ signals have been detected only a few times. It seems that the suppression of the low-frequency parasitics gave more space for the excitation of the higher-frequency signals.

\section{Configuration III: Stacked beam tunnel with optimized absorbers and ceramic-loaded spacer}

In order to verify the assumption that the remaining parasitic signals with frequencies higher than $151 \mathrm{GHz}$, which could be detected during the experiments with the standard (configuration I) as well as with the beam tunnel containing the optimized ceramic rings (configuration II), are excited in the area of the spacer, the smooth metallic spacer was (almost completely) replaced by additional stacked metallic and ceramic rings made from the same optimized low-permittivity material. The achieved performance using the ceramic-loaded spacer and an average beam current $I_{\mathrm{b}}=47 \mathrm{~A}$ is summarized in Fig. 8a. The results are normalized to $1.08 \mathrm{MW}$, achieved at $\left(\varphi_{\mathrm{B}}, R_{\mathrm{b}}\right)=\left(-3^{\circ}, 9.45 \mathrm{~mm}\right)$ with total efficiency $28 \%$. It is worthwhile to mention that all parasitic oscillations have been successfully suppressed, which confirms the assumption that the signals with frequencies $151 \mathrm{GHz}-159 \mathrm{GHz}$ are, indeed, excited in the area of the metallic spacer.

Fig. $8 \mathrm{~b}$ presents the power of the gyrotron when the average beam current is increased to $I_{\mathrm{b}}=54 \mathrm{~A}$. The results are normalized to the maximum power achieved at the operating point $\left(\varphi_{\mathrm{B}}, R_{\mathrm{b}}\right)=\left(-3^{\circ}, 9.55 \mathrm{~mm}\right)$, i.e. $1.22 \mathrm{MW}$ with total efficiency $27 \%$. By comparing Fig. $8 \mathrm{~b}$ with Fig. $7 \mathrm{~b}$ we notice that although the signals with frequencies above $151 \mathrm{GHz}$ are not completely suppressed (in contrast to the low-current case presented in Fig. 8a), they appear for a lower number of operating points. The high-power operation of the gyrotron has essentially retained its size with the peak power having increased despite the slightly lower beam current than the one used for the measurements in Fig. 7b.

The improved performance of the beam tunnel with the dielectric-loaded spacer becomes more evident by measuring the starting current of the parasitic signal that appears at each operating point. In order to do that, we progressively increase the beam current and we search for parasitic signals after we
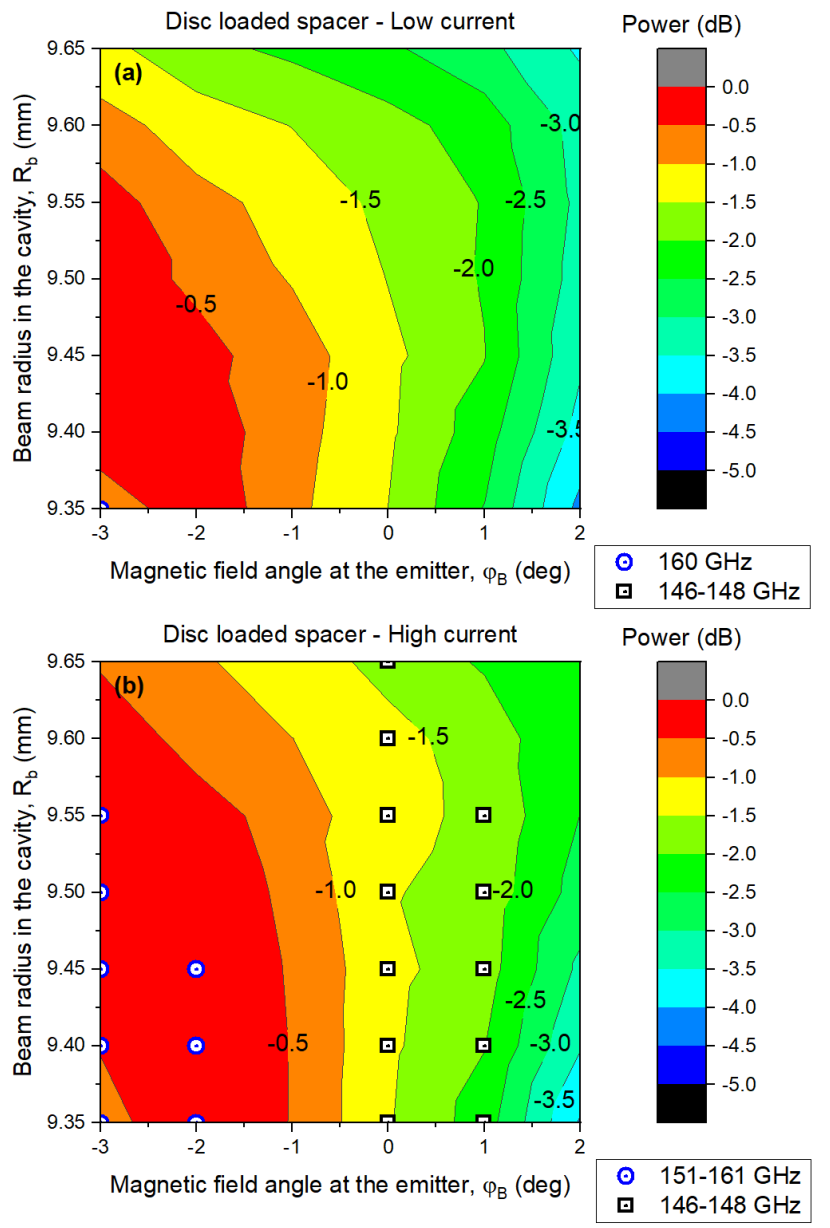

Fig. 8. Optimized stacked beam tunnel with additional dielectric rings in the spacer area. Output RF power with respect to the magnetic field angle $\varphi_{\mathrm{B}}$ at the emitter and the beam radius $R_{\mathrm{b}}$ in the cavity for (a) low (47 A) and (b) high beam current (54 A).

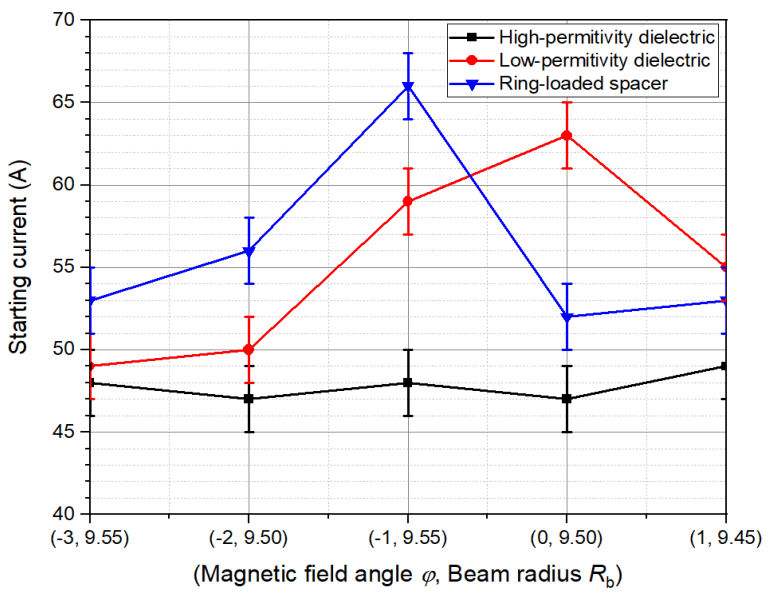

Fig. 9. Comparison of the parasitic signal starting currents for selected operating points.

optimize the accelerating voltage in terms of maximizing the output power. Fig. 9 presents the starting current for a selection of operating points that cover a wide range of magnetic field angles for beam radius values near $9.5 \mathrm{~mm}$. In the same figure, the corresponding starting currents for the beam tunnels with the low-permittivity ceramics (configuration II) and the high-permittivity ceramics (configuration I) are also visible. It is evident that the starting 
current of the case with the beam tunnel extended towards the spacer (configuration III) for the negative angles has been noticeably increased, approximately $10 \%$ compared to the beam tunnel with the high-permittivity ceramic rings. Essentially, we can achieve parasitic-free operation for any $\left(\varphi_{\mathrm{B}}, R_{\mathrm{b}}\right)$ combination up to $52 \mathrm{~A}$.

As a side effect of the spacer-area modification, the parasitic signals with frequencies in the $146 \mathrm{GHz}-148 \mathrm{GHz}$ range have reappeared. This supports further the assumption that the $146 \mathrm{GHz}-148 \mathrm{GHz}$ signals are excited very close to the last ceramic rings of the beam tunnel and by extending the stacked structure towards the cavity, we have actually increased the interaction length for these modes. The decrease of the corresponding starting currents compared to the beam tunnel with the metallic spacer (and the low-permittivity ceramic rings) can easily be seen in Fig. 9. It should be noted that even with this side effect, the starting currents of the parasitic signals are still higher compared to the standard baseline beam tunnel (high-permittivity ceramic rings and metallic spacer). Considering also that the gyrotron is always operated for negative magnetic field angles where the pitch factor is relatively high, minimizing or removing the smooth spacer region is the optimal choice in order to ensure parasiticfree operation with currents as high as $52 \mathrm{~A}$.

\section{EfFEct of the PARAsitic Signals on the RF POWER}

Although Fig. 5, Fig. 7 and Fig. 8 give an overview of the gyrotron performance with the different beam tunnels, they do not provide insight on how the parasitic signals affect the RF power. Fig. 10 presents the power and the corresponding total efficiency with respect to the beam current for selected operating points. As shown in Fig. 10a for the operating point $\left(-2^{\circ}, 9.50 \mathrm{~mm}\right)$ the generated power is increasing linearly with the beam current in the parasitics-free operating range. For beam currents higher than $56 \mathrm{~A}$, where the $151 \mathrm{GHz}$ $159 \mathrm{GHz}$ signals are detected (shaded area in Fig. 10a), the power has the tendency to saturate with the beam current, whereas the total efficiency drops. Similarly, for the operating point $\left(-1^{\circ}, 9.55 \mathrm{~mm}\right)$, where no parasitic signals have been detected for beam currents below $65 \mathrm{~A}$, the power is increasing almost linearly for beam current values up to $60 \mathrm{~A}$ (Fig. 10b). The power saturation and the efficiency drop that is observed higher than $60 \mathrm{~A}$ could be a strong indication that the beam current is approaching the starting current of the parasitic signal, which is confirmed by the fact that for the maximum current value that was tested, it was possible to detect a parasitic signal at $146 \mathrm{GHz}$ during the last $10 \mu \mathrm{s}$ of the pulse. Both effects, the saturation of the power and the reduction of the efficiency, can be attributed to the fact that the excitation of the parasitic signals increases the energy and the velocity spread of the electrons prior the main interaction in the cavity and as a result the electronic efficiency in the cavity degrades.

In practice, however, an additional phenomenon can contribute to the saturation of the power at higher beam currents. The modular gyrotron that has been used for the
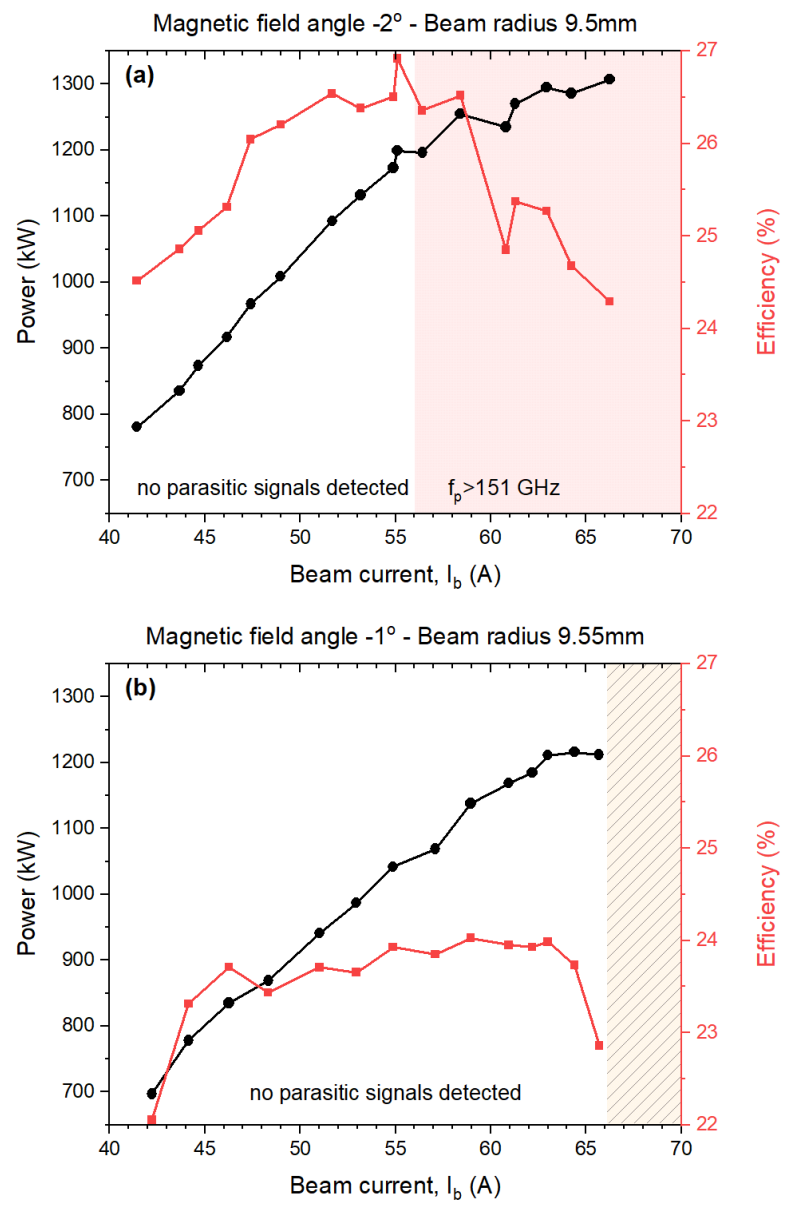

Fig. 10. Output power with respect to the beam current for the operating points (a) $\left(-2^{\circ}, 9.50 \mathrm{~mm}\right)$ and $(\mathrm{b})\left(-1^{\circ}, 9.55 \mathrm{~mm}\right)$.

experiments exhibits a relatively fast neutralization rate and consequently the beam voltage is increasing during the pulse. For this reason, the accelerating voltage is optimized in order to ensure that, despite the increase of the beam voltage during the pulse ( $1 \mathrm{~ms}$ flattop), a mode switch before the end of the pulse will not take place. Of course the neutralization rate depends on the vacuum quality as well as on the beam current. As the beam current increases, the rest gas in the tube is ionized faster and the neutralization rate increases. In turn, the accelerating voltage cannot follow the increase of the beam current in order to stay in the hard excitation area of the mode and sometimes is even necessary to slightly reduce it to avoid a mode switch. Both the increase of the beam current (higher voltage depression in the cavity) and the reduction of the accelerating voltage (lower pitch factor and lower detuning) could contribute to saturation of the output power. Considering that the excitation of RF signals in the beam tunnel could deteriorate the background vacuum closer to the cavity, it is possible that the excitation of the parasitics also speeds up the neutralization procedure.

Summarizing, the excitation of the parasitic signals could contribute to the saturation of the output power directly by increasing the electron's energy and velocity spread as well as indirectly by increasing the neutralization rate during the pulse. However, in short-pulse conditions it is difficult to define in what extent the parasitic oscillations deteriorate the 
main interaction in the cavity and which of the above described mechanisms has a stronger effect.

\section{CONCLUSION}

In the present work the nature of the parasitic oscillations observed in a high-power short-pulse gyrotron in the frequency range $145-161 \mathrm{GHz}$ has been experimentally studied by modifying the gyrotron beam-compression region.

The experimental results suggest that for all observed signals the parasitic interaction takes place before the main interaction and, in particular, at the compression zone closer to the cavity, where the modifications have been applied. Since these modifications have affected directly the starting current of all observed signals without a significant effect on the main interaction in the cavity, we can exclude that the parasitic signals are due to a dynamic after-cavity interaction as assumed for other high-power gyrotrons in the past [9]. In addition, the theoretical results of [13] have been confirmed, since the optimized low-permittivity, low-loss-tangent ceramic material led to higher starting currents of the parasitic signals, which for some operating points have been increased by about $40 \%$ giving a parasitic free operation with beam currents up to $60 \mathrm{~A}$ and higher. Finally, for the parasitic modes that were not affected by the optimized ceramics, it was shown, by extending the stacked ceramic-loaded section that they originate from an interaction at the area of the smooth metallic spacer confirming the findings of [22]. The final configuration led to an increase of the parasitic-modes starting currents for all operating points by more than $10 \%$.

Note that the enhanced suppression of the parasitic modes demonstrated in this work was achieved only by the optimized ceramic material. Careful selection of the geometric properties of the beam tunnel can lead to further increase of the overall losses, resulting in higher starting currents of the parasitics. This may be useful for conventional gyrotrons operating with high beam currents such as the $140 \mathrm{GHz}-1.5 \mathrm{MW}$ upgrade gyrotron for the W7-X stellarator [23].

\section{REFERENCES}

[1] M. K. A. Thumm, G. G. Denisov, K. Sakamoto and M.Q. Tran, "Highpower gyrotrons for electron cyclotron heating and current drive", Nuclear Fusion, vol. 59, no. 7, pp. 073001, June 2019, doi: doi.org/10.1088/1741-4326/ab2005.

[2] G. Gantenbein et al., "Experimental results of series gyrotrons for the stellarator W7-X," 22 $2^{\text {nd }}$ IAEA Fusion Energy Conference, Geneva, October 13-18, 2008. FT/P2-24.

[3] K. Felch, M. Blank, P. Borchard, P. Cahalan, S. Cauffman and H. Jory, "Recent test results on a $95 \mathrm{GHz}, 2$ MW Gyrotron," 2008 33rd International Conference on Infrared, Millimeter and Terahertz Waves, Pasadena, CA, 2008, pp. 1-2, doi: 10.1109/ICIMW.2008.4665499.

[4] K. Sakamoto, A. Kasugai, Y. Ikeda, K. Hayashi, K. takahasi, S. Moriyama, M. Seki, T. Kariya, Y. Mitsunaka, T. Fujii, T. Imai, "Development of 170 and $110 \mathrm{GHz}$ gyrotrons for fusion devices", Nuclear Fusion, vol. 43, no. 8, pp. 729-737, Aug 2003, doi: 10.1088/0029-5515/43/8/314.

[5] I. I. Antakov, I. G. Gachev and E. V. Zasypkin, "Self-excitation of spurious oscillations in the drift region of gyrotrons and their influence on gyrotron operation," in IEEE Transactions on Plasma Science, vol. 22, no. 5, pp. 878-882, Oct. 1994, doi: 10.1109/27.338303.
[6] M. Pedrozzi, S. Alberti, J. P. Hogge, M. Q. Tran, and T. M. Tran, "Electron beam instabilities in gyrotron beam tunnels," Physics of Plasmas, vol. 5, no. 6, pp. 2421-2430, Jun. 1998, doi: 10.1063/1.872918.

[7] A. Litvak, K. Sakamoto, and M. Thumm, "Innovation on high-power long-pulse gyrotrons," Plasma Physics and Controlled Fusion, vol. 53, no. 12, p. 124002, Nov. 2011, doi: 10.1088/0741-3335/53/12/124002.

[8] Z. C. Ioannidis et al., "Recent experiments with the European 1MW, $170 \mathrm{GHz}$ industrial $\mathrm{CW}$ and short-pulse gyrotrons for ITER," Fusion Engineering and Design, vol. 146, pp. 349-352, Sep. 2019, doi: 10.1016/j.fusengdes.2018.12.065.

[9] G. Gantenbein et al., "Experimental investigations and analysis of parasitic RF oscillations in high-power gyrotrons," IEEE Trans. Plasma Sci., vol. 38, no. 6, pp. 1168-1177, Jun. 2010, doi:10.1109/TPS.2010.2041366.

[10] G. P. Latsas, Z. C. Ioannidis, and I. G. Tigelis, "Dependence of Parasitic Modes on Geometry and Attenuation in Gyrotron Beam Tunnels," IEEE Transactions on Plasma Science, vol. 40, no. 6, pp. 1538-1544, Jun. 2012, doi: 10.1109/tps.2012.2192294.

[11] M. D. Moraitou, G. P. Latsas, Z. C. Ioannidis, and I. G. Tigelis, "Parasitic Oscillations in Coaxial Gyrotron Beam Tunnels," IEEE Transactions on Electron Devices, vol. 60, no. 4, pp. 1469-1475, Apr. 2013, doi: 10.1109/ted.2013.2247608.

[12] I. G. Chelis, K. A. Avramidis and J. L. Vomvoridis, "Resonant Modes of Disk-Loaded Cylindrical Structures With Open Boundaries," in IEEE Transactions on Microwave Theory and Techniques, vol. 63, no. 6, pp. 1781-1790, June 2015, doi: 10.1109/TMTT.2015.2420093.

[13] I. G. Chelis, K. A. Avramidis, Z. C. Ioannidis and I. G. Tigelis, "Improved Suppression of Parasitic Oscillations in Gyrotron Beam Tunnels by Proper Selection of the Lossy Ceramic Material," in IEEE Transactions on Electron Devices, vol. 65, no. 6, pp. 2301-2307, June 2018, doi:10.1109/TED.2017.2784198.

[14] T. Rzesnicki et al., "Recent experimental results of the European $1 \mathrm{MW}$, $170 \mathrm{GHz}$ short-pulse gyrotron prototype for ITER," 40th International Conference on Infrared, Millimeter, and Terahertz waves, Hong Kong, 2015, doi: 10.1109/IRMMW-THz.2015.7327492.

[15] I. G. Pagonakis et al., "Status of the development of the EU $170 \mathrm{GHz} / 1$ MW/CW gyrotron," Fusion Engineering and Design, vol. 96-97, pp. 149-154, Oct. 2015, doi: 10.1016/j.fusengdes.2015.02.050.

[16] H. O. Prinz, "Design and developrnent of a broadband real-time 100 $175 \mathrm{GHz}$ frequency measuremnent systen for gyrotron diagnostics," EUROCON 2005 - The International Conference on "Computer as a Tool", Belgrade, 2005, pp. 1871-1874, doi: 10.1109/EURCON.2005.1630346.

[17] A. Schlaich, G. Gantenbein, J. Jelonnek and M. Thumm, "Transient Millimeter-Wave Signal Analysis With Unambiguous RF Spectrum Reconstruction," in IEEE Transactions on Microwave Theory and Techniques, vol. 61, no. 12, pp. 4660-4666, Dec. 2013, doi: 10.1109/TMTT.2013.2283063.

[18] K. A. Avramidis et al., "A comparative study on the modeling of dynamic after-cavity interaction in gyrotrons," Physics of Plasmas, vol. 22, no. 5, p. 53106, May 2015, doi: 10.1063/1.4919924.

[19] I. G. Pagonakis, K. A. Avramidis, G. Gantenbein, T. Rzesnicki, A. Samartsev, and J. Jelonnek, "Magnetic field profile analysis for gyrotron experimental investigation," Physics of Plasmas, vol. 24, no. 3, p. 33102, Mar. 2017, doi: 10.1063/1.4977460.

[20] Y. Wang, X. Shang, N. M. Ridler, T. Huang, and W. Wu, "Characterization of Dielectric Materials at WR-15 Band (50-75 GHz) Using VNA-Based Technique," IEEE Transactions on Instrumentation and Measurement, vol. 69, no. 7, pp. 4930-4939, Jul. 2020, doi: 10.1109/tim.2019.2954010.

[21] Z. C. Ioannidis et al., "Development and Experimental Verification of an XY-Table for the Optimization of the Alignment of High-Power Gyrotrons," in IEEE Transactions on Electron Devices, vol. 66, no. 4, pp. 1954-1959, April 2019, doi: 10.1109/TED.2019.2899163.

[22] J. Genoud et al., "Parasitic Oscillations in Smooth-Wall Circular Symmetric Gyrotron Beam Ducts," Journal of Infrared, Millimeter, and Terahertz Waves, vol. 40, no. 2, pp. 131-149, Oct. 2018, doi: 10.1007/s10762-018-0548-5.

[23] K. A. Avramidis et al., "Studies towards an upgraded 1.5 MW gyrotron for W7-X," EPJ Web of Conferences, vol. 203, p. 4003, 2019, doi: 10.1051/epjconf/201920304003. 